Annuaire suisse de politique de développement

Société de l'information et coopération internationale

\title{
Pain pour le prochain : pour une communication équitable
}

\section{Christoph Stückelberger}

\section{(2) OpenEdition}

1 Journals

Édition électronique

URL : http://journals.openedition.org/aspd/571

DOI : 10.4000/aspd.571

ISSN : 1663-9669

Éditeur

Institut de hautes études internationales et du développement

Édition imprimée

Date de publication : 1 novembre 2003

Pagination : 145-147

ISSN : 1660-5934

\section{Référence électronique}

Christoph Stückelberger, "Pain pour le prochain : pour une communication équitable », Annuaire suisse de politique de développement [En ligne], 22-2 | 2003, mis en ligne le 22 mars 2010, consulté le 08 septembre 2020. URL : http://journals.openedition.org/aspd/571 ; DOI : https://doi.org/10.4000/aspd. 571 


\title{
Pain pour le prochain:
}

\section{pour une communication équitable}

\author{
Christoph Stückelberger*
}

\section{Une vision réaliste}

Un label Max Havelaar apposé non seulement sur le café et le miel, mais également sur des téléphones mobiles fabriqués dans des conditions équitables; un code de conduite tel que celui de la campagne Clean Clothes pour des «vêtements propres», mais cette fois-ci pour des articles de presse rédigés dans des conditions honnêtes et loyales; une liste noire des sociétés de télécommunications privatisées qui ont versé de généreux pots-de-vin aux ministres concernés afin d'acquérir un marché; des «tomates» pour les organes de presse qui ont perdu tout sens critique et se sont précipités dans la propagande belliciste des partisans de la guerre en Irak; un label d'entreprise pour les éditeurs sérieux; un label Max Havelaar pour un groupe de télécommunications qui, dans sa politique de prix, privilégie les liaisons dans les zones rurales et les pays pauvres en percevant un léger supplément sur les lignes rentables entre les grandes villes.

Ce qui vaut pour les produits alimentaires et les textiles vaut également pour l'information et la communication: un «commerce» plus équitable, qu'il s'agisse de technologies de la communication ou de contenus d'in- formation. Des codes de conduite et des chartes existent pour éditeurs et journalistes qui peuvent être améliorés. Les critères définissant des conditions de travail et des prix équitables dans la fabrication de téléphones mobiles et d'ordinateurs ne sont pas fondamentalement différents de ceux auxquels est soumise la fabrication d'un T-shirt ou de jus d'orange. Dans le domaine de l'information et de la communication, un contrôle indépendant et crédible (monitoring et vérification) représente là aussi une tâche exigeante et un défi considérable. Il pourrait stimuler dans ce domaine une concurrence positive pour des normes éthiques.

\section{La communication équitable participe de l'«économie équitable »}

Un «commerce équitable de l'information» peut éveiller la conscience que l'information et la communication sont des biens précieux, vitaux, qu'elles représentent plus qu'une marchandise. La communication équitable fait partie de l' «économie équitable». Celle-ci, les relations économiques justes, constitue la priorité de Pain pour le prochain (PPP) dans la politique de développement. Ce n'est donc pas un hasard, mais le fruit d'une logique interne, si PPP tra-

* Secrétaire général de Pain pour le prochain et professeur d'éthique à la Faculté de théologie de l'Université de Bâle. 
vaille sur cette question dans cinq domaines, interdépendants: le commerce de biens et services, les échanges financiers (placements éthiques, désendettement), l'information et la communication (TIC), la lutte contre la corruption et la promotion d'une éthique économique dans les pays du Sud. Il s'agit là d'éléments essentiels constitutifs d'un ensemble de relations équitables. A cet égard, PPP souligne que l'accès à l'information et à la communication doit, comme pour les autres biens, être équitable. Par ailleurs, l'information et la communication sont plus que des marchandises. De nombreux développements d'ordre culturel, éthique, religieux ou social sont touchés par l'information et la communication et doivent donc être pris en compte. PPP, notamment son secrétaire général en tant qu'éthicien, s'engage en faveur de l'éthique dans les médias. Le Sommet mondial sur la société de l'information en décembre 2003 ne devra pas aborder les TIC sous le seul angle technique, mais en étudier aussi les implications sociales.

\section{Les TIC}

\section{au service du développement}

Les projets de développement montrent bien l'ambiguïté des TIC: par la concentration de pouvoir qu'elles induisent ou leur usage abusif, elles peuvent accroître la fracture numérique, aggraver le fossé entre pauvres et riches. Mais elles peuvent aussi encourager la formation de communautés, soutenir d'une manière essentielle les efforts de communication et de lutte contre la pauvreté, augmenter la participation démocratique et la transparence, au service de la paix.
C'est dans ce sens seulement que les TIC peuvent être l'instrument d'un développement juste, durable, pacifique et participatif. PPP, depuis un quart de siècle, s'est engagé dans cette voie.

\section{Des projets de communication depuis plus de vingt-cing ans}

Pain pour le prochain, le service des Eglises protestantes suisses pour le développement, s'est engagé depuis 1975 dans des projets de développement touchant à l'information et à la communication. Hans Ott, longtemps secrétaire général de PPP, avait soutenu à l'époque différents projets d'information: production de radiocassettes à Taiwan, lancement du quotidien Wantok («one talk») en Papouasie-Nouvelle-Guinée ou encore formation de journalistes et installation d'émetteurs radio en Afrique. Ces projets ont été menés en coopération avec l'EPER et des missions telles que l'ancienne Mission de Bâle, mais également avec l'Association mondiale pour la communication chrétienne (AMCC/WACC). Hans Ott a siégé au comité international de l'AMCC du milieu des années 1970 au milieu des années 1980. Outre le soutien de projets médias, qui s'est poursuivi sans interruption jusqu'à aujourd'hui, PPP a participé depuis le début des années 1980 au débat de politique de développement sur le nouvel ordre mondial de la communication, avant et après la publication du fameux rapport McBride. Cela, par exemple, avec les études de Hans Ott Sind Satelliten Entwicklungshelfer? et d'Urs A. Jaeggi Kein Interesse an einer neuen Weltkommunikationsordnung ${ }^{1}$. 
Retour des nouvelles technologies sur le devant de la scène

Ayant concentré ses efforts en matière de politique de développement sur les questions de commerce équitable, PPP avait, dans les années 1990, relégué quelque peu au second plan les problèmes de communication, sans pour autant abandonner les projets déjà lancés dans ce domaine. Mais à la fin de 1999, en coopération avec l'Action de Carême, elle a ramené les nouvelles technologies de l'information et de la communication (NTIC) sur le devant de la scène en les plaçant au cœur de la campagne œcuménique 2002. Le thème, «Partager la communication», a notamment servi au lancement d'une pétition, adressée à la Confédération, aux sociétés de télécommunications et aux journalistes/éditeurs, réclamant la «communication pour tous». «La communication est un droit humain. L'accès aux moyens de communication est un facteur clé du développement économique et social des populations de pays défavorisés.» $\mathrm{Au}$ travers de cette revendication centrale, la coopération suisse au développement était invitée concrètement à se montrer plus généreuse, de façon à permettre un meilleur accès des pays en développement aux technologies de la communication; les sociétés de télécommunications ont été priées de prendre en compte, dans leur politique tarifaire, les régions économiquement faibles en Suisse et à l'étranger; les médias, enfin, ont été appelés à adapter leur ligne éditoriale à la «Charte populaire de la communication». 